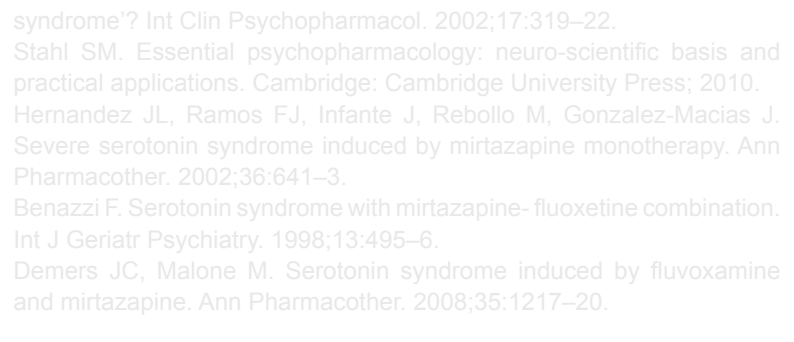

\title{
Desafios Diagnósticos e Terapêuticos na Síndrome Hemolítica Urémica Atípica: A Propósito de Um Caso Clínico
}

\section{Diagnostic and Therapeutic Challenges in Atypical Hemolytic Uremic Syndrome: A Case Report}

\author{
Sofia REIS $\triangle^{1}$, Daniela RAMOS ${ }^{2}$, Carolina CORDINHÃ ${ }^{3}$, Clara GOMES ${ }^{3}$
}

Acta Med Port 2019 Oct;32(10):673-675 - https://doi.org/10.20344/amp.10021

\begin{abstract}
RESUMO
A síndrome hemolítica urémica atípica constitui uma microangiopatia trombótica resultante da hiperativação da via alterna do complemento. A sua gravidade exige diagnóstico e terapêutica precoces. O diagnóstico diferencial inclui a síndrome hemolítica urémica típica (associada à toxina Shiga) e a púrpura trombótica trombocitopénica (associada a deficiência na atividade da ADAMTS13). A terapêutica específica da síndrome hemolítica urémica atípica, atualmente disponível, é o eculizumab. Descrevemos um caso clínico de uma criança com síndrome hemolítica urémica atípica diagnosticada em contexto de diarreia sanguinolenta, complicada de envolvimento neurológico e que colocou vários desafios no diagnóstico e decisões terapêuticas.
\end{abstract}

Palavras-chave: Eculizumab; Síndrome Hemolítica Urémica Atípica

\section{ABSTRACT}

The atypical hemolytic uremic syndrome comprises a thrombotic microangiopathy resulting from the complement alternate pathway hyperactivation. Its severity requires early diagnosis and treatment. The differential diagnosis includes typical hemolytic uremic syndrome (associated with Shiga toxin) and thrombotic thrombocytopenic purpura (associated with deficient activity of ADAMTS13). The only specific treatment currently available for atypical hemolytic uremic syndrome is eculizumab. We describe the case of a child with atypical hemolytic uremic syndrome diagnosed in the context of bloody diarrhea, complicated by neurological involvement that posed several diagnostic and therapeutic challenges.

Keywords: Atypical Hemolytic Uremic Syndrome; Eculizumab

\section{INTRODUÇÃO}

A síndrome hemolítica urémica (SHU) define-se por lesão renal aguda, trombocitopenia e anemia hemolítica microangiopática. ${ }^{1-3}$

A causa mais comum da SHU é a toxina Shiga $(90 \%$ dos casos pediátricos), produzida, habitualmente, pelo serotipo 0157:H7 da Escherichia coli, definindo a SHU típica. ${ }^{1,4,5}$ O diagnóstico confirma-se com a identificação da bactéria em coprocultura, da toxina por imunoensaios e polimerase chain reaction (PCR) e/ou pela pesquisa de anticorpos no soro. ${ }^{4} \mathrm{O}$ diagnóstico diferencial inclui a púrpura trombótica trombocitopénica caracterizada pela deficiência na atividade da ADAMTS13 (metaloprotease que cliva os multímeros do fator de Von Willebrand). ${ }^{4}$
A SHU atípica (SHUa) resulta da hiperativação da via alterna do complemento. Estima-se uma prevalência pediátrica de 7/1 $000000 .{ }^{1}$ Pode determinar isquemia em vários órgãos, sendo o rim e cérebro os mais afetados, seguidos do trato gastrointestinal e coração. As manifestações extra-renais ocorrem em $20 \%$ dos doentes. ${ }^{3,4}$ Pode ser primária ou secundária a doença auto-imune, transplante, fármacos, gestação, sépsis, hipertensão maligna e tumores. ${ }^{4} \mathrm{~A}$ forma primária deve-se a mutações nos genes de proteínas da via alterna do complemento ( $50 \%-60 \%$ dos doentes); as mais frequentes afetam o fator $\mathrm{H}(20 \%-30 \%)$, 5,6 sendo também descritas mutações na proteína do cofator de membrana CD46, fator I, trombomodulina, fatores ativadores do

1. Serviço de Pediatria. Centro Hospitalar Tondela-Viseu. Viseu. Portugal.

2. Hospital Pediátrico. Centro Hospitalar e Universitário de Coimbra. Coimbra. Portugal.

3. Unidade de Nefrologia Pediátrica. Hospital Pediátrico de Coimbra. Centro Hospitalar e Universitário de Coimbra. Coimbra. Portugal.

$\triangle$ Autor correspondente: Sofia Reis. reis.carlasofia@gmail.com

Recebido: 07 de dezembro de 2017 - 18 de outubro de 2018 | Copyright $\odot$ Ordem dos Médicos 2019 
fator B e C3 e ainda autoanticorpos para o fator $\mathrm{H}^{5} \mathrm{Em}$ $80 \%$ dos casos as mutações são esporádicas. Na SHUa secundária podem ser identificadas mutações ou polimorfismos de risco, que se manifestam apenas perante outro evento desencadeante. ${ }^{4}$

A SHUa tinha mau prognóstico com evolução para doença renal crónica terminal e mortalidade elevada em idade pediátrica. ${ }^{1} \mathrm{O}$ eculizumab, um anticorpo monoclonal, com afinidade para a proteína C5 do complemento e que bloqueia a formação de C5a e do complexo C5b-9 suprimindo a atividade microangiopática trombótica, surgiu como uma terapêutica especifica do SHUa. ${ }^{1,2,4,5}$

Como a SHUa se associa ao risco de infeção por Neisseria meningitidis, cuja eliminação é dependente do complexo C5b-9, é obrigatória a vacinação (meningococos A, C, W, Y e B) duas semanas antes do início do tratamento ou antibioterapia profilática se período inferior. ${ }^{7-9}$ Outros efeitos adversos são hipertensão arterial, infeções respiratórias, diarreia, vómitos e cefaleias. ${ }^{10}$

Os autores descrevem os desafios no diagnóstico e nas decisões terapêuticas de uma criança com SHUa.

\section{CASO CLÍNICO}

Menina de oito anos, saudável, com diarreia muco-sanguinolenta e dor abdominal, que no D8 de queixas referiu aparecimento de hematúria. Não apresentava contexto epidemiológico de doença. Encontrava-se pálida e anictérica. Analiticamente destacava-se redução de $2 \mathrm{~g} / \mathrm{dL}$ na hemoglobina em 36 horas (de 12,3 para 10,3 g/dL), 14 000/uL plaquetas, esfregaço de sangue periférico com acantócitos e esquizócitos, ureia $102 \mathrm{mg} / \mathrm{dL}$, creatinina $1,3 \mathrm{mg} / \mathrm{dL}$ e desidrogenase láctica 4055 U/L.

Foi transferida para hospital nível III por suspeita de SHU. A avaliação analítica era sobreponível com haptoglobina $<0,07 \mathrm{~g} / \mathrm{L}$, doseamento da atividade ADAMTS13 normal e PCR de bactérias produtoras de toxina Shiga em zaragatoa retal negativa. A ecografia mostrou rins globosos e perda da diferenciação corticomedular. Foi colocado o diagnóstico de SHUa e, por indisponibilidade imediata de eculizumab, iniciou plasmaferese.

O estudo de autoimunidade, PCR para o vírus influenza $\mathrm{A}$ e $\mathrm{B}, \mathrm{VIH} 1$ e 2, vírus da hepatite $\mathrm{B}(\mathrm{VHB})$ e vírus da hepatite $\mathrm{C}(\mathrm{VHC})$, hemocultura e coprocultura foram negativos.

No D3 de internamento, após terceira sessão de plasmaferese, apresentou alteração súbita de consciência, desvio conjugado do olhar para a direita, hemianópsia homónima e hemiparésia esquerda. A tomografia axial crânio-encefálica não mostrou alterações. O ecocardiograma revelou trombo na ponta do catéter venoso central (CVC) na aurícula direita e estrutura filamentosa na veia cava inferior, aorta ascendente e crossa, não identificando forâmen oval patente (FOP). O eletroencefalograma mostrou lentificação global da atividade elétrica e a ressonância magnética áreas de hipersinal no córtex temporal, parassagital e parieto-occipital à direita, algumas atingindo a substância branca subcortical adjacente, compatíveis com lesões de isquémia/enfarte. Iniciou enoxaparina subcutânea, manteve plasmaferese em novo acesso vascular. Pela impossibilidade de se excluir um fenómeno microangiopático insistiu-se na disponibilização urgente do eculizumab.

Por convulsão focal à direita (D7) iniciou fenitoína que manteve até D21.

Houve normalização da função renal em D10 de internamento, recuperação da hemoglobina e normalização das plaquetas em D14, tendo realizado 12 sessões de plasmaferese, até D13. Em D17 iniciou eculizumab $(20 \mathrm{mg} /$ $\mathrm{kg} /$ dose) e amoxicilina profilática após pesquisa negativa de Neisseria meningitidis na orofaringe, imunização com vacinas meningocócica $B, A, C, W 135$ e Y e pneumocócica polivalente 23 .

Teve alta ao fim de 21 dias de internamento, clinicamente bem. Cumpriu três administrações de eculizumab, com intervalo semanal, sem intercorrências.

Entretanto foi conhecido o estudo genético do complemento que não identificou mutações nem polimorfismos de risco.

Perante este resultado e a recuperação, mantida após suspensão da plasmaferese, foi decidido descontinuar o tratamento com eculizumab.

Seis meses depois estava neurologicamente bem e apresentava ressonância magnética sem lesões, tendo suspenso enoxaparina.

Aos oito meses de seguimento mantinha-se assintomática, sem evidência de atividade microangiopática.

\section{DISCUSSÃO}

Perante um quadro de microangiopatia trombótica deve dosear-se a ADAMTS13 e pesquisar a toxina Shiga. Se ambas forem normais o diagnóstico provável é SHUa.

A terapêutica específica do SHUa é o eculizumab, idealmente iniciado nas primeiras $24-48$ horas da doença. ${ }^{9}$ Enquanto não está disponível, pelo processo burocrático de autorização, a alternativa é a plasmaferese. ${ }^{9}$

O evento neurológico nesta criança foi o fator decisivo para iniciar eculizumab. Apesar de ter sido identificado trombo no CVC não havia FOP. O envolvimento cerebral ocorre em $8 \%-30 \%$ dos casos de SHU $^{10}$ mas pode ser uma complicação da plasmaferese por trombose do CVC e o diagnóstico diferencial não é fácil.

A suspensão do eculizumab é controversa. É uma terapêutica dispendiosa, tem riscos ${ }^{4,6,9}$ mas a descontinuação pode condicionar reagravamento da microangiopatia e complicar-se de manifestações renais, neurológicas e cardiovasculares. ${ }^{6}$

Nesta criança suspendeu-se o eculizumab atendendo à melhoria mantida após a interrupção da plasmaferese e estudo genético normal. ${ }^{5}$

Como em cerca de metade dos casos não são identificadas alterações genéticas, a sua ausência não exclui a possibilidade de recidiva da microangiopatia, imprevisível na gravidade e no tempo, e a necessidade de recomeçar o eculizumab, ${ }^{11}$ pelo que a doente tem indicação para avaliação analítica nas intercorrências agudas. ${ }^{5,9}$

Permanece o debate quanto à etiologia e terapêutica do 
SHUa sem mutações identificáveis. ${ }^{10}$ Surgiram novos fármacos dirigidos a moléculas da via do complemento como o fator $\mathrm{H}, \mathrm{C} 3$ e C3/C5 convertase mas ainda sem utilização clínica. ${ }^{10} \mathrm{O}$ eculizumab constitui a terapêutica de primeira linha e a sua disponibilização precoce e menos burocratizada nos hospitais de nível III é fundamental.

\section{AGRADECIMENTOS}

Os autores agradecem a Carmen do Carmo, da Unidade de Nefrologia Pediátrica do Hospital Pediátrico - Centro Hospitalar e Universitário de Coimbra, a colaboração na orientação do caso clínico supracitado e na revisão do presente artigo.

\section{PROTEÇÃO DE PESSOAS E ANIMAIS}

Os autores declaram que os procedimentos seguidos estavam de acordo com os regulamentos estabelecidos pelos responsáveis da Comissão de Investigação Clínica

\section{REFERÊNCIAS}

1. Rathbone J, Kaltenthaler E, Richards A, Tappenden P, Bessey A, Cantrell A. A systematic review of eculizumab for atypical haemolytic uraemic syndrome (aHUS). BMJ Open. 2013;3:e003573.

2. Legendre CM, Licht C, Muus P, Greenbaum LA, Babu S, Bedrosian C, et al. Terminal complement inhibitor eculizumab in atypical hemolyticuremic syndrome. N Engl J Med. 2013;368:2169-81.

3. Zhang K, Lu Y, Harley KT, Tran MH. Atypical hemolytic uremic syndrome: a brief review. Hematol Rep. 2017;9:7053.

4. Vaisbich MH. Síndrome hemolítico-urêmica na infância. J Bras Nefrol. 2014;36:208-20.

5. Choo AZ, Brown F. Subclinical atypical haemolytic uremic syndrome relapse following discontinuation of eculizumab. Nephrology. 2017;1:4-6.

6. Fakouri F, Fila M, Provôt F, Delmas Y, Barbet C, Châtelet V, et al. Pathogenic variants in complement genes and risk of atypical hemolytic uremic syndrome relapse after eculizumab discontinuation. Clin J Am Soc Nephrol. 2017;12:50-9.

7. McNamara LA, Wang X, Hariri S, Fox L, MacNeil JR. High Risk for e Ética e de acordo com a Declaração de Helsínquia da Associação Médica Mundial.

\section{CONFIDENCIALIDADE DOS DADOS}

Os autores declaram ter seguido os protocolos do seu centro de trabalho acerca da publicação de dados.

\section{CONFLITOS DE INTERESSE}

Os autores declaram não ter conflitos de interesses relacionados com o presente trabalho.

\section{CONSENTIMENTO PARENTAL}

Obtido.

\section{FONTES DE FINANCIAMENTO}

Este trabalho não recebeu qualquer tipo de suporte financeiro de nenhuma entidade no domínio público ou privado.

invasive meningococcal disease among patients receiving eculizumab (soliris) despite receipt of meningococcal vaccine. MMWR Morb Mortal Wkly Rep. 2017;66:734-7.

8. Cohn AC, MacNeil JR, Clark TA, Ortega-Sanchez IR, Briere EZ, Meissner $\mathrm{HC}$, et al. Prevention and control of meningococcal disease: recommendations of the advisory committee on immunization practices. Recommendations and reports. 2013/62(RR02);1-22 [consultado 2017 nov 28]. Disponível em: https://www.cdc.gov/mmwr/preview/mmwrhtml/ rr6202a1.htm.

9. Loirat C, Fakhouri F, Ariceta G, Besbas N, Bitzan M, Bjerre A, et al. An international consensus approach to the management of atypical hemolytic uremic syndrome in children. Pediatr Nephrol. 2016;31:15-39.

10. Verhave JC, Wetzels JF, Van de Kar CA. Novel aspects of atypical haemolytic uraemic syndrome and the role of eculizumab. Nephrol Dial Transplant. 2014;29:131-41.

11. Macia M, Moreno FA, Dutt T, Fehrman I, Hadaya K, Gasteyger C, et al. Current evidence on the discontinuation of eculizumab in patients with atypical haemolytic uraemic syndrome. Clin Kidney J. 2017;10:310-9. 